\title{
Nascent Weighted Page Rank Algorithm
}

\author{
Balwant Kumar \\ Department of CSE, GJUS\&T, \\ Hisar, Haryana-125001, India
}

\author{
Dharmender Kumar \\ Department of CSE, GJUS\&T, \\ Hisar, Haryana-125001, India
}

\begin{abstract}
There may be a millions of web pages that include a particular words or specific phrases. However some of them will be more relevant and popular than others. Modern search engines apply methods of ranking the results to present the best results first after that just plain text searching. The main objective of this paper is to explain the various existing page ranking algorithms and the enhancement done to the standard page rank algorithm. The weighted page rank algorithm based on visits of links by user is enhanced and a new algorithm called Nascent Weighted Page Rank (NWPR) algorithm is proposed. The proposed algorithm considers the additional factor of weight due to outlinking pages in spite of weight due to inlinking pages and the visits of links by user in calculating the page rank. It is observed that the results of the proposed algorithm are comparable to the previously known algorithms. Also the value of page ranks of web pages computed by the NWPR is largely dependent on the value of $d$ (damping factor).
\end{abstract}

\section{Keywords}

Page Rank, Weighted Page Rank, NWPR, Inlinks, Outlinks.

\section{INTRODUCTION}

With the advancement in engineering and technology now-adays internet is an important part of our everyday lives and information is just a click away from us. Page Rank is broadly used for ranking web pages in order of relevance by mostly all search engines world-wide. A few search engine uses link structure based page ranking algorithm while other uses content based or usage based page ranking algorithm [2]. The page ranking algorithm shows the popularity of a web page in its page rank score. But with the thriving requirements of ordering more and more relevant web pages, the conventional page rank algorithm undergoes several improvements and enhancements. Page Rank is broadly used for ranking web pages in order of relevance by mostly all search engines world-wide [3]. There are several algorithms for page ranking such as Google Page Rank algorithm, (HITS) HyperlinkInduced Topic Search algorithm, Weighted Page Rank algorithm [1] etc. There are basically three categories of page ranking algorithm namely, link analysis algorithm, personalized web search ranking algorithm [13] and page segmentation algorithm.

- Link structure analysis algorithm: The link structure analysis algorithm is based on the linking structure of the web pages. The number of inlinks or outlinks to a web page are mainly the input parameters used to calculate the rank value of that page.

- Personalized web search ranking algorithm: Personalization is the process of gathering all the experience of individual user. The main aim of the personalization was to deliver the more satisfied results to the end user by giving most relevant information.
- Page Segmentation algorithm: Page segmentation algorithms are used to partition full web page into many smaller blocks. Because of some special characteristic features of web pages such as the varying length of the web pages and also multiple topics, various page segmentation approaches have influenced the performance of the web searching process.

There are basically 4 categories of different page segmentation algorithm as follows [8]:

- Fixed length Page segmentation

- DOM based page segmentation

- Vision based page segmentation

- Combined approach segmentation

There are many algorithms for link structure based page ranking such as Google Page Rank algorithm, Weighted Page Rank algorithm, (HITS) Hyperlink-Induced Topic Search algorithm etc. [11]. Now let us discuss about the background related works in page ranking algorithms.

\section{EXISTING ALGORITHMS}

There are many algorithms for link structure based page ranking such as Google Page Rank algorithm, Weighted Page Rank algorithm, (HITS) Hyperlink-Induced Topic Search algorithm etc.[3,4,7,9]. A few search engine uses link structure based page ranking algorithm [4] while other uses usage and content based. Now various page ranking algorithms based on link structure analysis are discussed, beginning with the standard (PGA) Page Rank algorithm.

\section{1 (GPRA) Google Page Rank Algorithm}

In 1998, Larry Page and Sergey Brin, the founders of google, invented the Page Rank Algorithm to quantize the significance of millions of web pages composing the (WWW) World Wide Web. Page Rank of a web page is a numerical number representing the significance of that web page based on the number of inbound links [10]. Page Rank algorithm is a link structure based algorithm. The basic idea of Page Rank is that the significance of a page is directly proportional to the number of web pages linking to that page. So Page Rank algorithm acknowledge a page more important if large number of other web pages are linking to that page or if links are coming from some of most essential and popular web pages. Page Rank of a web page $X$ is determined by the page rank of those pages that links to page $\mathrm{X}$ using formula given below:

Where,

$$
P R(X)=(1-d)+d\left[\frac{P R\left(Y_{1}\right)}{C\left(Y_{1}\right)}+\ldots \ldots+\frac{P R\left(Y_{n}\right)}{C\left(Y_{n}\right)}\right]
$$

PR $\left(Y_{i}\right)=$ Page Rank of pages $Y_{i}$ that links to a web page $X$

PR $(X)=$ Page Rank of web page $X$,

$\mathrm{d}=$ Damping Factor (value between 0 and 1, but generally its value is 0.85 ) 
$\mathrm{C}\left(\mathrm{Y}_{\mathrm{i}}\right)=$ Number of outbound links on web page $\mathrm{Y}_{\mathrm{i}}$ Rerun this above step involving the computation of page rank until two successive same values are obtained.

\section{Advantages of Page Rank:}

- The algorithm being robust against Spam because it is not as easy for an owner to add some inlinks to his web page from other important pages.

- $\quad$ Page Rank being a global measure is query independent.

Disadvantages of Page Rank:

- The main disadvantage of Page Rank is that it mainly favoured the older pages, because a new web page, even a good one is not having many links unless it has become a part of an existing website.

- $\quad$ Page Rank could be easily increased by using the concept of "link-farms". But, while indexing, the search tries to find these flaws actively.

(PGA) Page Rank algorithm used by search engine shows the web page according to their page rank. Page Rank algorithm is non-keyword specific [9]. The major drawback of page rank algorithm is that it is calculated and stored at the indexing time and not at the time of query. The (SERP) Search Engine Ranking Positions of any website bank upon the actual current status of that website whereas Page Rank determined by using Page Ranking algorithm depends upon the stored database which is amend only once in a period of two-three months. The Page Rank Algorithm divides the page rank value uniformly to all the out linking web pages but all outlinks are not equally significant or relevant to user query. This problem can be solved by seeing an additional weight factor [8] in the calculation of page rank and gives birth to a new algorithm known as Weighted Page Rank algorithm.

\subsection{Weighted Page Rank Algorithm}

Wenpu Xing and Ali Ghorbani [1] have proposed a page ranking algorithm called weighted page rank (WPR). This algorithm is basically an extension to the Google Page Rank algorithm. Weighted Page Rank has taken into account the importance from both the number of inlinks and outlinks of the pages and distributed the page rank scores based upon the popularity of the web pages. WPR performed better than the previous Page Ranking algorithm in terms of returning factor and it will return larger number of relevant pages to a given user query. According to the basic idea, the more prominent webpages are the more linkages that other webpages tend to have to them or are linked to by them. The proposed approach extended Page Rank algorithm to a Weighted Page Rank Algorithm that assign larger rank values to most important pages instead of dividing the rank value of a page equally among all its outlinking pages. This (WPRG) weighted page rank [7] algorithm is different from the conventional page rank algorithm in the fact that each outlink page has a page rank value proportional to its importance (i.e. number of inlinking pages and number of outlinking pages).

$\mathrm{W}^{\mathrm{in}}(\mathrm{v}, \mathrm{u})$, the popularity or importance from the number of inlinks, can be calculated depending upon the number of inlinking pages of page $u$ and the number of inlinking pages of all reference pages of page $\mathrm{v}$.

$\mathrm{W}^{\mathrm{in}}(\mathrm{v}, \mathrm{u})=$ weight of link $(\mathrm{v}, \mathrm{u})$ or importance of web page due to inlinks

$$
W_{(v, u)}^{i n}=\frac{I_{u}}{\sum_{p \in R(v)} I_{p}}
$$

Where, $I_{p}$ and $I_{u}$ denote the total number of inlinks of page $p$ and page $\mathrm{u}$ respectively, $\mathrm{R}(\mathrm{v})$ denotes the list of the reference pages of page $\mathrm{v}$.

$\mathrm{W}^{\text {out }}(\mathrm{v}, \mathrm{u})$, the popularity or importance from the number of outlinks, is calculated based upon the number of outlinking pages of page $u$ and the number of outlinking pages of all reference pages of page $v$.

$\mathrm{W}^{\text {out }}(\mathrm{v}, \mathrm{u})=$ weight of link $(\mathrm{v}, \mathrm{u})$ or importance of web page due to outlinks

$$
W_{(v, u)}^{\text {out }}=\frac{O_{u}}{\sum_{p \in R(v)} O_{p}}
$$

Where, $\mathrm{O}_{\mathrm{p}}$ and $\mathrm{O}_{\mathrm{u}}$ denote the number of outlinking pages of page $\mathrm{p}$ and page $\mathrm{u}$ respectively, $\mathrm{R}(\mathrm{v})$ denotes the list of the reference pages of page $\mathrm{v}$.

After considering the importance of web pages, the modified page rank formula is given as [4]:

$$
P R(u)=(1-d)+d \sum_{v \in B(u)} P R(v) W_{(v, u)}^{\text {in }} W_{(v, u)}^{\text {out }}
$$

This (WPRA) Weighted Page Rank algorithm determine the problem of ranking web pages based on their relevancy or importance by considering the weight factor. But the problem of query independency and calculation of page ranks at indexing time still remain with WPR and with the traditional Page Ranking algorithm.

\subsection{Page Rank Based on Visits of Links}

G. Kumar and N. Duhan [5] et al. proposed a page ranking algorithm based on visits of links. This algorithm worked on the basic ranking algorithm of Google i.e. Page Rank and took number of visits of inbound links of Web pages into account. The modified version based on VOL is given in the following equation:

$$
P R(u)=(1-d)+d \sum_{v \in B(u)} \frac{L_{\mathrm{u}} \mathrm{PR}(\mathrm{v})}{T L(v)}
$$

Notations are:

- u represents a web page,

- d is Damping Factor,

- $\quad B(u)$ is the set of pages that point to $u$,

- $\mathrm{L}_{\mathrm{u}}$ is the number of visits for the links which are pointing page $\mathrm{u}$ from $\mathrm{v}$.

- $\quad \mathrm{PR}(\mathrm{u})$ and $\mathrm{PR}(\mathrm{v})$ are rank scores of page $\mathrm{u}$ and $\mathrm{v}$ respectively,.

- $\quad$ TL (v) denotes total number of visits of all links present on $\mathrm{v}$.

The proposed approach diverges from the common trend by taking the additional factor of the number of visits to the inbound links of page. To calculate the hits or visits of an outgoing links on a web page, a client side script was used. Whenever a web page was accessed the script will be loaded on client side from the web server. Script would take care of the click as well as keyboard event to occur [12]. When an event occur and if that event will happen over hyperlink then it will send a message to web server with information of current web page and hyperlink. On server side a database of 
$\log$ file will be used to record the web page id, hyperlinks of that page and hit count of hyperlinks. Hit count would incremented every time a hit occur on hyperlink. This hit count would be stored in search engine's database which was used to calculate the rank value of different web pages or documents. Larger the rank value of web page means that page was commonly visited by users. Hence the method displays the most important pages based on the user's interest and minimizes the search space. The proposed approach gave a method to find link-visit counts of Web pages and a comparison between VOL with the Page Rank algorithm.

\subsection{Weighted Page Rank based on VOL}

N. Tyagi and S. Sharma [6] proposed an algorithm called Weighted Page Rank Algorithm based on the probability of visits of links. The proposed algorithm was an extension to Weighted Page Rank Algorithm and made comparison between original Weighted Page Rank method and Visits of Links method. The original WPR algorithm assigned page rank value to each outlink page directly proportional to its popularity. But more was the number of visits to a links by user; more is the importance of web page. The advanced approach in the new algorithm was to determine the user's usage trends. The user's browsing behaviour could be calculated by number of hits (visits) of links.

This modified algorithm calculates PageRank value or importance of web pages based on the visits of incoming links on a page. It does not consider here the popularity of outlinks which is considered in the original algorithm.

$$
W P R_{\mathrm{vol}}(u)=(1-d)+d \sum_{v \in B(u)} \frac{L_{\mathrm{u}} W P R_{\mathrm{vol}}(v) W_{(\mathrm{v}, \mathrm{u})}^{\mathrm{in}}}{T L(v)}
$$

Notations are:

- d is Damping Factor,

- $\mathrm{u}$ represents a web page,

- $\quad B(u)$ is the set of pages that point to $u$,

- $\mathrm{WPR}_{\mathrm{VOL}}(\mathrm{u})$ and $\mathrm{WPR}_{\mathrm{VOL}}(\mathrm{v})$ are rank scores of page $u$ and $v$ respectively,

- $\mathrm{L}_{\mathrm{u}}$ is the number of visits for links which are pointing page $\mathrm{u}$ from $\mathrm{v}$.

- $\quad$ TL (v) denotes total number of visits to all linking pages present on $\mathrm{v}$.

$\mathrm{W}^{\mathrm{in}}(\mathrm{v}, \mathrm{u})=$ weight of link $(\mathrm{v}, \mathrm{u})$ or importance of web page due to inlinks

$$
W_{(v, u)}^{i n}=\frac{I_{u}}{\sum_{p \in R(v)} I_{p}}
$$

Where, $I_{u}$ and $I_{p}$ denote the no. of inlinks of page $u$ and page $p$ respectively, $R(v)$ represents the reference page list of page $v$. The proposed algorithm consider that the most visited outlinks have higher rank value and thus higher popularity. In this approach, a user cannot willingly increase the rank of a page by visiting the page multiple times because the rank of the page depends on the probability of visits (not on the count of visits) on back linked pages.

\section{PROPOSED ALGORITHM}

The proposed work includes a new algorithm called Nascent Weighted Page Rank Algorithm (NWPR) for calculating the page rank scores of web pages. In the proposed algorithm, the concept of Weighted Page Rank and Weighted Page Rank Algorithm based on Visit of links (VOL) is extended. The both inlinks and outlinks of a web page contribute to some extent in computing the importance of a web page. Therefore we have used an additional outlinks weight in addition to inlinks weight used in WPR (VOL). In WPR based on VOL algorithm, only the popularity of inlinks is considered. But it does not consider the popularity of outlinks. And in the proposed algorithm we are considering both the popularity of outlinks and inlinks. The page rank values using original WPR remained unaffected whether the web pages are being accessed by the user or not, that is the relevancy of a web page not being taken into account. But the page rank values by proposed method assigns higher ranks to web pages having more number of visits of links. This concept of using popularity of inlinks and outlinks helps to improve the convergence point. The NWPR algorithm also gives comparable results in relation to other existing algorithms. Let's know about the different steps of NWPR algorithm.

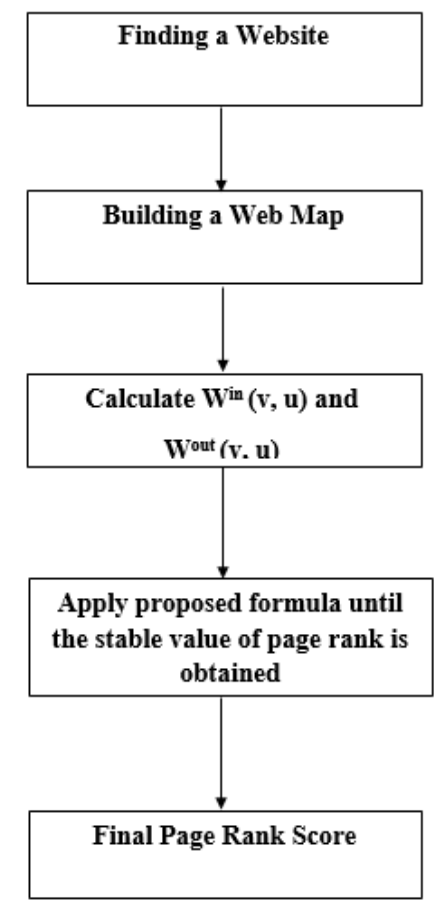

Fig.1: Flow Chart of NWPR Algorithm

The various steps involved in the proposed algorithm are explained as follows:

Step 1: Finding a good web site

This step involves finding a website having many hyperlinks as the weighted Page Rank and WPR (VOL) methods depends on the link based web structures. We consider the link structure of web site to calculate the page rank in NWPR algorithm. The rich hyperlinked website is good for testing the working of the proposed algorithm.

Step 2: Generate a web graph

This step involves the generation of the web graph from the selected website. This web graph had date or information about the inlinks and outlinks of a page.

Step 3: Calculate the number of visits or hits of hyperlinks Client side script was used to monitor the counts or hits of hyperlinks and information was then sent to the web server and finally information was accessed by crawlers. 
Step 4: Calculating the page rank of each web page Then calculate the $\mathrm{W}^{\text {in }}(\mathrm{v}, \mathrm{u})$ and $\mathrm{W}^{\text {out }}(\mathrm{v}, \mathrm{u})$ for each node present in the web graph after applying the equation (4.1) and (4.2). $\mathrm{W}^{\mathrm{in}}(\mathrm{v}, \mathrm{u})=$ weight of link $(\mathrm{v}, \mathrm{u})$ or importance of a web page due to its inlinks

$$
W_{(v, u)}^{i n}=\frac{I_{u}}{\sum_{p \in R(v)} I_{p}}
$$

where, $I_{p}$ and $I_{u}$ denote the no. of inlinks of page $p$ and page $u$ respectively, $\mathrm{R}(\mathrm{v})$ denotes the list of reference pages of page $\mathrm{v} . \mathrm{W}^{\mathrm{in}}(\mathrm{v}, \mathrm{u})$, the popularity due to the number of inlinks and is computed based on number of inlinking pages of page $u$ and number of inlinking pages of all referenced pages of page $\mathrm{v}$. $\mathrm{W}^{\text {out }}(\mathrm{v}, \mathrm{u})$, the popularity due to the number of outlinking pages and is computed based on the number of outlinking pages of page $u$ and the number of outlinking pages of all referenced pages of page $v$.

$\mathrm{W}^{\text {out }}(\mathrm{v}, \mathrm{u})=$ weight of link $(\mathrm{v}, \mathrm{u})$ or importance of web page due to its outlinks

$$
W_{(v, u)}^{\text {out }}=\frac{O_{u}}{\sum_{p \in R(v)} O_{p}}
$$

Where, $\mathrm{O}_{\mathrm{p}}$ and $\mathrm{O}_{\mathrm{u}}$ denote the no. of outlinks of page $\mathrm{p}$ and page $u$ respectively, $R(v)$ denotes the list of reference page of page v.

\section{Step 5: Applying the proposed formula}

This step involves the calculation of the Page Rank values of the nodes present in the web graph by making use of the proposed formula. $\mathrm{W}^{\text {in }}(\mathrm{v}, \mathrm{u})$ and $\mathrm{W}^{\text {out }}(\mathrm{v}, \mathrm{u})$ values are being used to calculate page rank from the modified equation (4.3):

$$
\begin{aligned}
& N W P R_{V O L}(u) \\
& =(1-d) \\
& +d \sum_{v \in B(u)} \frac{L_{u} N W P R_{V O L}(v) W_{(v, u)}^{\text {in }} W_{(v, u)}^{\text {out }}}{T L(v)}
\end{aligned}
$$

Notations are:

- d is Damping Factor,

- u denotes a web page,

- $\quad \mathrm{B}(\mathrm{u})$ denotes the set of all pages pointing to $\mathrm{u}$,

- $\quad \mathrm{NWPR}_{\mathrm{VOL}}(\mathrm{u})$ and $\mathrm{NWPR}_{\mathrm{VOL}}(\mathrm{v})$ are rank values of web pages $u$ and $v$ respectively,

- $\mathrm{L}_{\mathrm{u}}$ denotes the number of visits of linking pages which are pointing to page $\mathrm{u}$ from $\mathrm{v}$.

- $\quad$ TL (v) is total no. of visits of all linking pages present on $\mathrm{v}$.

\section{Step 6: Recursively going to Step 4}

The last step is used recursively until the values comes out to be stable. And the algorithm will converge when we get two equal values of page ranks in two consecutive repetitions.

To explain the working of proposed Nascent Weighted PageRank (NWPR) algorithm, let us take an example of the hyperlinked structure shown in figure 4.2, we have demonstrated a small web graph consisting of 3 pages A, B, C. Here webpage $\mathrm{A}$ is linking to webpage $\mathrm{B}$ and $\mathrm{C}$, webpage $\mathrm{B}$ is linking to webpage $\mathrm{C}$ and webpage $\mathrm{C}$ is linking to webpage A and every link is having its own corresponding visits.

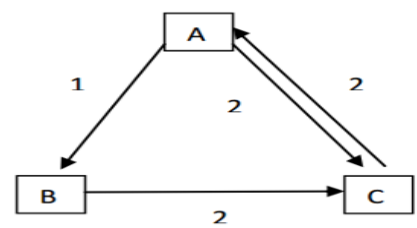

Fig 2: Hyper linked web graph of A, B, C pages with link visits

The Nascent Weighted PageRank (NWPR) algorithm calculates the page rank for pages $\mathrm{A}, \mathrm{B}$ and $\mathrm{C}$ by using equations as given below:

$$
\begin{gathered}
W_{(C, A)}^{\text {in }}=\frac{I_{A}}{I_{A}+I_{B}}=\frac{1}{1+1}=\frac{1}{2} \\
W_{(C, A)}^{\text {out }}=\frac{O_{A}}{O_{A}+O_{B}}=\frac{2}{2+1}=\frac{2}{3} \\
W_{(A, B)}^{\text {in }}=\frac{I_{B}}{I_{C}}=\frac{1}{2} \quad W_{(A, B)}^{\text {out }}=\frac{O_{B}}{O_{C}}=\frac{1}{1} \\
W_{(A, C)}^{\text {in }}=\frac{I_{C}}{I_{C}}=\frac{2}{2}=1 \quad W_{(A, C)}^{\text {out }}=\frac{O_{C}}{O_{C}}=\frac{1}{1}=1 \\
W_{(B, C)}^{\text {in }}=\frac{I_{C}}{I_{A}}=\frac{2}{1}=2 \quad W_{(B, C)}^{\text {out }}=\frac{O_{C}}{O_{A}}=\frac{1}{2} \\
\frac{L_{A}}{T L(C)}=\frac{2}{2}=1 \quad \frac{L_{B}}{T L(A)}=\frac{1}{3} \\
\frac{L_{C}}{T L(A)}=\frac{2}{3} \quad \frac{L_{C}}{T L(B)}=\frac{2}{2}=1
\end{gathered}
$$

Now these values are used to compute final page rank of A, B, $\mathrm{C}$ using the following equations:

$$
\begin{aligned}
& N W P R_{V O L}(A)= \\
& (1-d)+ \\
& d\left(\frac{N W P R_{V O L}(C) W_{(C, A)}^{\text {in }} W_{(C, A)}^{\text {out }} L_{A}}{T L(C)}\right) \\
& N W P R_{V O L}(B)= \\
& (1-d)+ \\
& d\left(\frac{N W P R_{V O L}(A) W_{(A, B)}^{\text {in }} W_{(A, B)}^{\text {out }} L_{B}}{T L(A)}\right) \\
& N W P R_{V O L}(C)= \\
& (1-d)+d\left(\frac{N W P R_{V O L}(A) W_{(A, C)}^{\text {in }} W_{(A, C)}^{\text {out }} L_{C}}{T L(A)}\right. \\
& \left.\quad+\frac{N W P R_{V O L}(B) W_{(B, C)}^{\text {in }} W_{(B, C)}^{\text {out }} L_{C}}{T L(B)}\right)
\end{aligned}
$$

After calculating the values of above equations, the final page rank score of different web pages in a web Graph are obtained. Equations for page ranks of pages in the web graph in figure 2 using NWPR algorithm can be written using equations (4.4), (4.5), (4.6) given in previous chapter as follows:

$\operatorname{PR}(\mathrm{A})=(1-\mathrm{d})+\mathrm{d}(\mathrm{PR}(\mathrm{C}) * 1 / 2 * 2 / 3)$

$\mathrm{PR}(\mathrm{B})=(1-\mathrm{d})+\mathrm{d}(\mathrm{PR}(\mathrm{A}) * 1 / 2 * 1 / 3)$ 
$\mathrm{PR}(\mathrm{C})=(1-\mathrm{d})+\mathrm{d}((\mathrm{PR}(\mathrm{A}) * 2 / 3)+(\mathrm{PR}(\mathrm{B})))$

After these calculations, we got different page rank values for each web page A, B, C. Now we apply the scaling of the page rank values such that the sum of rank of all the pages is 1 . The formula used for scaling is as follows:

$\mathrm{TPR}=\mathrm{PR}(\mathrm{A})+\mathrm{PR}(\mathrm{B})+\mathrm{PR}(\mathrm{C})$

$\mathrm{PR}(\mathrm{A})=\mathrm{PR}(\mathrm{A}) / \mathrm{TPR}$

$\mathrm{PR}(\mathrm{B})=\mathrm{PR}(\mathrm{B}) / \mathrm{TPR}$

$\mathrm{PR}(\mathrm{C})=\mathrm{PR}(\mathrm{C}) / \mathrm{TPR}$

The Scaled values of page rank computed by applying different values of $d$ (damping factor) in the NWPR algorithm. We have calculated the page rank scores of NWPR algorithm

Table 1: Page rank values of various algorithms at $\mathrm{d}=\mathbf{0 . 8 5}$

\begin{tabular}{|c|c|c|c|c|}
\hline $\begin{array}{c}\text { Web } \\
\text { Page } \\
\text { No. }\end{array}$ & $\begin{array}{c}\text { Page } \\
\text { Rank }\end{array}$ & WPR & $\begin{array}{c}\text { WPR } \\
\text { (VOL) }\end{array}$ & NWPR \\
\hline A & 0.3974 & 0.31779 & 0.3248 & 0.2999 \\
\hline B & 0.2148 & 0.16612 & 0.1451 & 0.2008 \\
\hline C & 0.3878 & 0.51608 & 0.5299 & 0.4991 \\
\hline
\end{tabular}

for value $\mathrm{d}=0.35, \mathrm{~d}=0.5$, and $\mathrm{d}=0.85$ as shown in Table 4 .

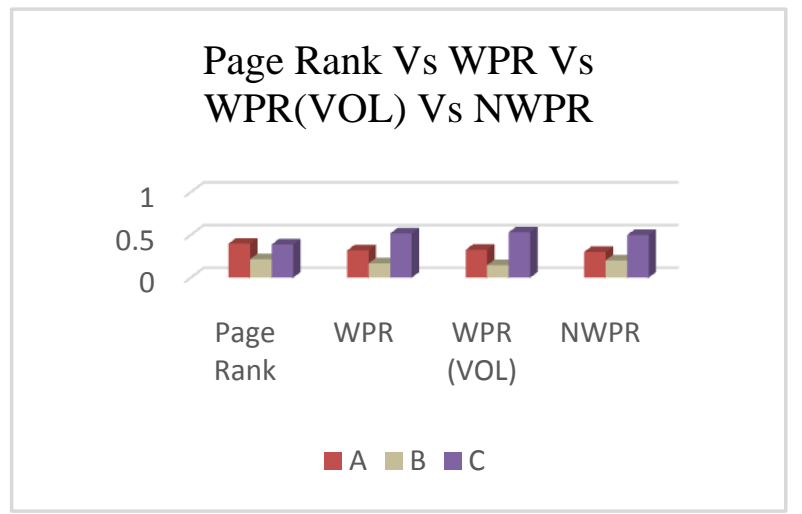

Fig 3: Variations for different ranking algorithms at $\mathrm{d}=\mathbf{0 . 8 5}$

Table 2: Page rank values of various algorithms at $\mathrm{d}=\mathbf{0 . 5}$

\begin{tabular}{|c|c|c|c|c|}
\hline $\begin{array}{c}\text { Web } \\
\text { Page } \\
\text { No. }\end{array}$ & $\begin{array}{c}\text { Page } \\
\text { Rank }\end{array}$ & WPR & $\begin{array}{c}\text { WPR } \\
\text { (VOL) }\end{array}$ & NWPR \\
\hline A & 0.3846 & 0.30183 & 0.30392 & 0.3 \\
\hline B & 0.2564 & 0.22007 & 0.20661 & 0.25 \\
\hline C & 0.359 & 0.4781 & 0.48947 & 0.45 \\
\hline
\end{tabular}

\section{Page Rank Vs WPR Vs \\ WPR(VOL) Vs NWPR}

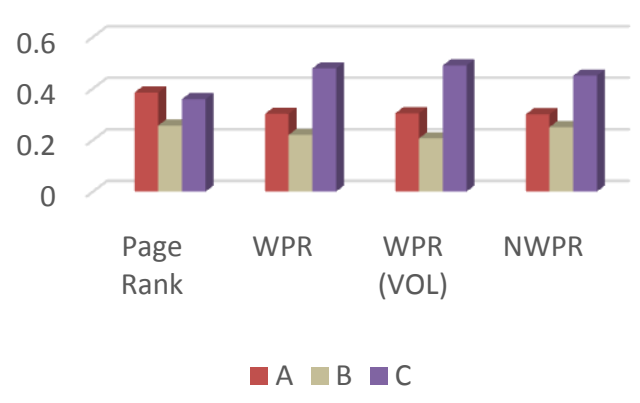

Fig 4: Variations for different ranking algorithms at $d=0.5$

Table 3: Page rank values of various algorithms at $\mathrm{d}=\mathbf{0 . 3 5}$

\begin{tabular}{|c|c|c|c|c|}
\hline $\begin{array}{c}\text { Web } \\
\text { Page } \\
\text { No. }\end{array}$ & $\begin{array}{c}\text { Page } \\
\text { Rank }\end{array}$ & WPR & $\begin{array}{c}\text { WPR } \\
\text { (VOL) }\end{array}$ & NWPR \\
\hline A & 0.3747 & 0.30154 & 0.30174 & 0.30467 \\
\hline B & 0.2775 & 0.24905 & 0.239 & 0.27319 \\
\hline C & 0.3478 & 0.44939 & 0.45925 & 0.42213 \\
\hline
\end{tabular}

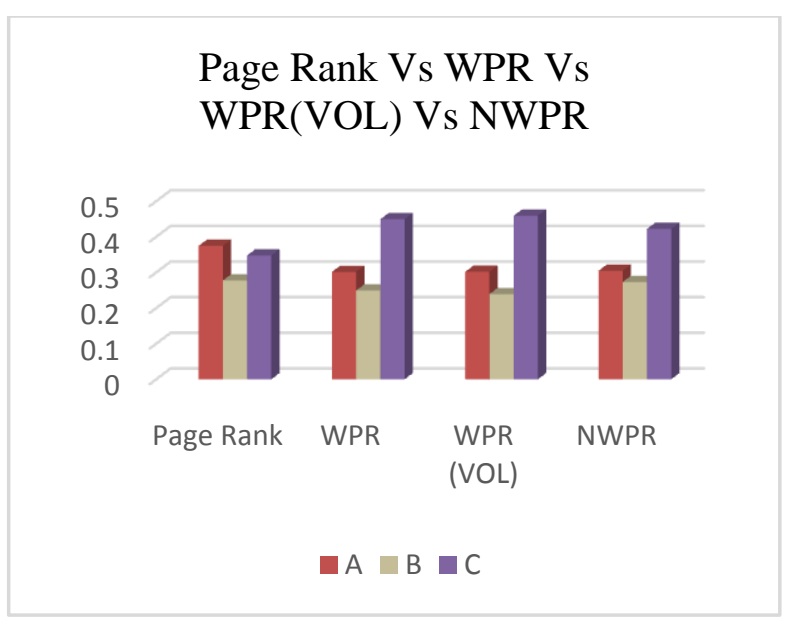

Fig 5: Variations for different ranking algorithms at $\mathrm{d}=\mathbf{0 . 3 5}$

Table 4: Page Rank Values of NWPR at different values of d

\begin{tabular}{|c|c|c|c|}
\hline $\begin{array}{c}\text { Web } \\
\text { Page } \\
\text { No. }\end{array}$ & $\begin{array}{c}\text { NWPR } \\
(\mathbf{d}=\mathbf{0 . 3 5})\end{array}$ & $\begin{array}{c}\text { NWPR } \\
(\mathbf{d}=\mathbf{0 . 5})\end{array}$ & $\begin{array}{c}\text { NWPR } \\
(\mathbf{d}=\mathbf{0 . 8 5})\end{array}$ \\
\hline $\mathrm{A}$ & 0.30467 & 0.3 & 0.2999 \\
\hline $\mathrm{B}$ & 0.27319 & 0.25 & 0.2008 \\
\hline $\mathrm{C}$ & 0.42213 & 0.45 & 0.4991 \\
\hline
\end{tabular}




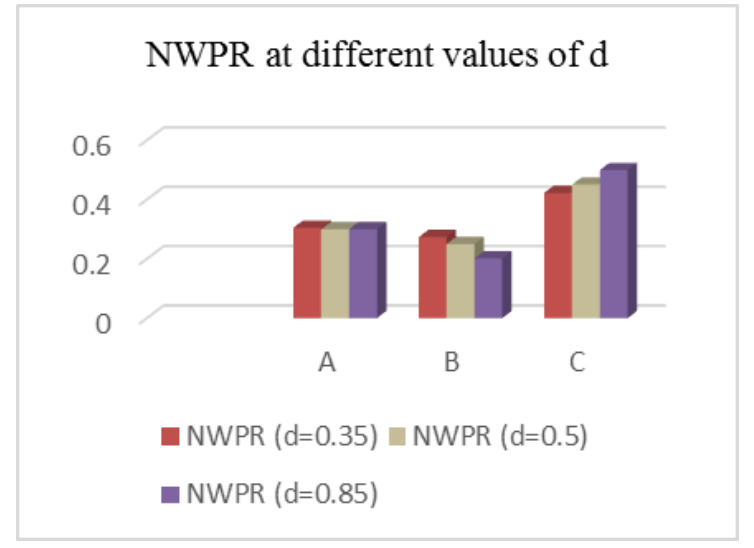

Fig 6: NWPR page rank values at different values of $d$

\section{RESULT ANALYSIS}

Now by comparing the page rank scores of webpages according to conventional Page Ranking algorithms it has been computed rank scores of every page corresponding to Page Rank algorithm, Weighted Page Rank (WPR), Weighted Page Rank using VOL and then proposed algorithm for the graph as shown in figure 2.The WPR basically uses web structure mining to compute the scores of page rank, whereas the proposed algorithm makes use of both mining techniques, namely web structure mining and additional web usage mining to compute scores of page rank. Also the WPR (VOL) normally uses popularity or importance only due to the number of in linking pages and not due to our linking pages. The proposed approach makes use of number of visits of in linking and out linking pages to calculate page rank and assigns more rank score to more popular or important web pages. The order of pages ranked according to page rank, WPR and WPR (VOL) is $\mathrm{B}<\mathrm{A}<\mathrm{C}$. The order of pages ranked according to the proposed NWPR algorithm is also $\mathrm{B}<\mathrm{A}<\mathrm{C}$. But the proposed algorithm converges faster than the WPR (VOL).The order of pages according to the rank score calculated using the NWPR algorithm is also same as that of standard page rank, WPR and WPR based on VOL. Page rank score for the proposed algorithm using different values of damping factor (d) have been calculated because the values of page ranks is largely dependent on the value of $d$. The table 4 shows the various values of page rank corresponding to different values of $d$ such as $d=0.35, d=0.5$ and $d=0.85$. Its graphical representation is shown in figure 6 using bar graph. The values for page rank $\mathrm{A}, \mathrm{B}$ and $\mathrm{C}$ corresponding to different values of $\mathrm{d}$ is different. For pages $\mathrm{A}$ and $\mathrm{B}$, on decreasing the values of $\mathrm{d}$, the page rank scores of $\mathrm{A}$ and $\mathrm{B}$ will increase. While on the other hand for the page $\mathrm{C}$, on increasing the values of $d$, the page rank score will increase. Hence for page rank of some pages the small value of $d$ is desirable, whereas for some pages the large value of $\mathrm{d}$ produces better results.

\section{CONCLUSION AND FUTURE SCOPE}

In this paper, the various algorithms and techniques mainly used by search engines in ranking web pages on the internet are studied. A modified page ranking algorithms called NWPR, which is more target oriented than other traditional weighted page rank algorithms is presented. This new proposed algorithm calculates the page rank score and importance of webpages depending on the visits of all the incoming links and outgoing links on a page. It not only considers link structure, but also includes the choice of different users while visiting a particular page. User normally has a habit of spending a lot of their time in surfing through all the search results displayed in order to find the most relevant webpages. We presented a novel page ranking algorithm called NWPR that provides more relevant results than original Weighted Page Rank. The ordering of pages in this way will increase the relevancy of pages and therefore provides the user with much better search results in terms of quality. Therefore, user may find its desired information or content webpages at the top of the list of web pages displayed by search engine, and in this way user's search space can be reduced. Other information such as, for example some kind of feedback from search engine asking about the information or list of webpages chosen by different users out of whole list displayed to them can be considered. But these enhancements are not sufficient to cope with the increasing data or information on every web page day-by-day. More experiments could be done with much bigger data sets in order to check the relevance of the proposed algorithm and prove that the proposed algorithm is comparably more convenient than that of the existing ones. The existing algorithms may consider the bookmarked web pages in calculating the Page Rank of web pages.

\section{REFERENCES}

[1] Wenpu Xing, A. Ghorbani. 2004. Weighted page rank algorithm. In Proceedings of Second Annual Conference, Communication Networks and Services Research. IEEE (2004),305-314.

[2] T. I. A. N Chong. 2010. A kind of algorithm for page ranking based on classified tree in search engine. In proceedings of International Conference on Computer Application and System Modeling (ICCASM). IEEE (2010) 13, 513-538.

[3] H. Dubey and B. N. Roy. 2011. An improved page rank algorithm based on optimized normalization technique. Int. J. Comp. Sci. and Info. Tech. 2183-2188.

[4] H. Ryang et al. 2014. Ranking algorithm for book reviews with user tendency and collective intelligence. Multi. Tools and App. 74 (May 2014), 1-19.

[5] G. Kumar et al. 2011. Page ranking based on number of visits of links of Web page. In Proceedings of $2^{\text {nd }}$ International Conference on Computer and Communication Technology (ICCCT). IEEE, 2011.

[6] N. Tyagi and S. Sharma. 2012. Weighted Page rank algorithm based on number of visits of Links of web page. Int. J. Soft Comp. and Eng. (IJSCE) 3 (July 2012), 387-391.

[7] M. Sehgal et al. 2014. Enhanced Page Rank Algorithm Using Time Factor. Int. J. Eng. and Comp. 3 (July 2014), 6990-6995.

[8] P. Patel. 2014. Research of Page ranking algorithm on search engine using Damping factor. Int. J. Ad. Eng. and Res. Dev. 1 (Feb. 2014), 1-6.

[9] Y. Qin and D. Xu. 2010. A Balanced Rank Algorithm based on page rank and page belief Recommendation. IEEE.

[10] S. Setayesh and A. Harounabadi et al. 2014. Presentation of an Extended Version of the Page Rank Algorithm to Rank Web Pages Inspired by Ant Colony Algorithm. Int. J. Comp. App. 85 (Jan. 2014), 975 - 8887. 
International Journal of Computer Applications (0975 - 8887) Volume 157 - No 3, January 2017

[11] M. Shamiul Amin et al. 2015. A score based web page ranking algorithm” Int. J. Comp. App. 110 (Jan. 2015), 11-15.

[12] A. Jain et al. 2013. Page ranking algorithm in web mining, limitations of existing methods and a new method for indexing web pages. In Proceedings of
International Conference on Communication Systems and Network Technologies. IEEE, 2013.

[13] T. S. Govada and N. L. Prasanna. 2014. Comparative study of various page ranking algorithms in web content mining. Int. J. of Ad. Res. 2 (July 2014), 457-464. 